\title{
Tunability enhancement of gene regulatory motifs through competition for regulatory protein resources
}

\author{
Swetamber Das* and Sandeep Choubey 1 \\ Max Planck Institute for the Physics of Complex Systems, Nöthnitzer Straße 38, 01187 Dresden, Germany
}

(Received 17 July 2020; accepted 12 October 2020; published 24 November 2020)

\begin{abstract}
Gene regulatory networks (GRNs) orchestrate the spatiotemporal levels of gene expression, thereby regulating various cellular functions ranging from embryonic development to tissue homeostasis. Some patterns called "motifs" recurrently appear in the GRNs. Owing to the prevalence of these motifs they have been subjected to much investigation, both in the context of understanding cellular decision making and engineering synthetic circuits. Mounting experimental evidence suggests that (1) the copy number of genes associated with these motifs varies, and (2) proteins produced from these genes bind to decoy binding sites on the genome as well as promoters driving the expression of other genes. Together, these two processes engender competition for protein resources within a cell. To unravel how competition for protein resources affects the dynamical properties of regulatory motifs, we propose a simple kinetic model that explicitly incorporates copy number variation $(\mathrm{CNV})$ of genes and decoy binding of proteins. Using quasi-steady-state approximations, we theoretically investigate the transient and steady-state properties of three of the commonly found motifs: Autoregulation, toggle switch, and repressilator. While protein resource competition alters the timescales to reach the steady state for all these motifs, the dynamical properties of the toggle switch and repressilator are affected in multiple ways. For toggle switch, the basins of attraction of the known attractors are dramatically altered if one set of proteins binds to decoys more frequently than the other, an effect which gets suppressed as the copy number of the toggle switch is enhanced. For repressilators, protein sharing leads to an emergence of oscillation in regions of parameter space that were previously nonoscillatory. Intriguingly, both the amplitude and frequency of oscillation are altered in a nonlinear manner through the interplay of CNV and decoy binding. Overall, competition for protein resources within a cell provides an additional layer of regulation of gene regulatory motifs.
\end{abstract}

DOI: 10.1103/PhysRevE.102.052410

\section{INTRODUCTION}

Gene regulatory networks (GRNs) consist of various molecular regulators such as transcription factors (TFs) that interact with each other to perform a repertoire of functions in cells, ranging from embryonic development to tissue homeostasis [1-3]. Intriguingly, certain subnetworks in the GRN are overrepresented. Such subnetworks are called motifs [4-6]. Due to the ubiquitous nature of gene regulatory motifs, a quantitative understanding of the dynamical behavior of them has concerned systems biologists and synthetic biologists alike [7-10]. While theorists have focused on mapping out the dynamic range of these circuits, experimentalists have sought to build them using a bottom-up approach. Such an engineering approach adds twofold value to the field; on the one hand, it furthers our understanding of the design

\footnotetext{
*Present address: Department of Chemistry, University of Massachusetts Boston, 100 Morrissey Boulevard, Boston, MA 02125, USA.

Published by the American Physical Society under the terms of the Creative Commons Attribution 4.0 International license. Further distribution of this work must maintain attribution to the author(s) and the published article's title, journal citation, and DOI. Open access publication funded by the Max Planck Society.
}

principles of gene regulatory motifs inside cells, and on the other, it helps in fabricating artificial circuits.

Most of these studies implicitly assume that gene regulatory motifs remain functionally isolated in a cell [11-13]. This assumption suffers from the complication that genetic motifs are always connected to many other genes inside a cell, which couples these motifs to various aspects of cell physiology. One such aspect is that protein products pertaining to genes of a motif bind promiscuously to a large number of decoy sites, as well as functional regulatory sites. Evidently, regulatory sites for a given gene may perform as decoy sites for another gene. For instance, well-known E.coli protein CRP has around 400 binding sites per genome copy [14]. The protein product of NF-kB, a gene associated with a eukaryotic oscillator, binds to a large number of sites across the genome; chip-seq data revealed approximately 20 thousand binding sites, with around 500 of them being functional [15-18]. Moreover, the copy number of genes within regulatory motifs can significantly vary in cells. For example, the copy number variation $(\mathrm{CNV})$ of genomes is widely prevalent in humans, as has been demonstrated in numerous studies [19-23]. Genes expressed on plasmids [24] or multiple identical copies on the chromosome [25-27], highly replicated viral DNA genes [28], etc. manifest key examples of CNV. Evolutionary analysis suggests that in some cases, whole-genome duplications led to lineage diversification in yeast [29]. The ubiquitous nature of $\mathrm{CNV}$ and decoy binding invokes competition for protein 
resources. A recent study explored the effect of gene copy number fluctuation [30] on the dynamical properties of wellknown regulatory motifs. On the other hand, several papers in the recent past have considered the effect of decoy binding $[5,12,18,31-35]$ in understanding the behavior of specific regulatory motifs. A quantitative understanding of how the interplay between $\mathrm{CNV}$ and decoy binding dictates the dynamical properties of gene regulatory motifs remains lacking.

To this end we dissect the dynamical properties of three well-known gene regulatory motifs, (i) the autoregulatory motif [36,37], (ii) the toggle switch [38-40], and (iii) repressilator $[7,10]$, in the presence of CNV and decoy binding. By utilizing the inherent separation of timescales of the system, we theoretically dissect this system [41]. Our investigation reveals that competition for finite protein resources alters the time to reach the steady state for all three motifs. Moreover, the toggle switch and repressilator motifs exhibit qualitatively distinct dynamical behaviors; the basins of attraction of a toggle switch is dramatically altered when one of the two sets of proteins bind to decoys more frequently. This alteration, though, is suppressed as the copy number of the toggle switch is ramped up. For repressilators, the interplay of decoy binding and copy number variation leads to either inhibition or abatement of oscillations, depending on the parameter space. Moreover, the impact of CNV and decoy binding is rather complex on the amplitude of oscillations; in a context-dependent manner, the amplitude can either increase or decrease. We discuss the relevance of these results in the context of development and synthetic biology.

\section{RESULTS}

To decipher the impact of CNV and decoy binding of protein products of genes pertaining to regulatory motifs on their dynamical properties, we consider three well-studied gene regulatory motifs - autoregulatory motif, toggle switch, and repressilator. In particular, we add $N_{p}$ copies of the motif under investigation and $N_{d}$ numbers of decoy binding sites in the system to study how the interplay of these two dictate the transient and steady-state behaviors of the motifs. We employ the so-called prefactor method, as developed earlier [41] and extend it in a congruous manner to achieve our goal. The analytical method is briefly illustrated in the next section; the details of the method are provided in Appendix A. Moreover, the parameter values chosen for the various models in our study lie in a range with experimental measurements in bacteria and viruses. (A list of all parameters used in numerical simulations can be found in the figure captions and in the Appendices.) In the following section, we start with the autoregulatory motif owing to its simplicity.

\section{A. Autoregulation}

Autoregulation is the simplest regulatory motif, which also happens to be ubiquitous in cells [36,37]. It comprises a single gene, the product of which regulates its own expression, as shown in Fig. 1(a). In this motif, the promoter transitions between two states which are defined by the promoter being bound and/or unbound to the protein; the rate of transition from the protein bound state to the empty promoter state is $k_{-}$while rate of switching from the empty promoter state to the protein bound state is $k_{+}$. When the promoter is proteinbound, mRNA production commences at a rate $\alpha$. The basal mRNA production rate from the empty promoter state is $\beta$. Each mRNA molecule is translated at a rate $\sigma$ which leads to monomeric protein production. Protein monomers reversibly bind to each other to form a dimer with rate $\kappa_{+}$which can subsequently dissociate into monomers at rate $\kappa_{-}$. mRNA and monomeric proteins degrade at rates $\gamma_{m}$ and $\gamma_{p}$, respectively. In our model, the dimeric proteins can bind to $N_{d}$ number of decoy sites in addition to $N_{p}$ number of promoters. We assume that the binding/unbinding rates of dimeric proteins to promoter and decoys are similar. Here it is assumed that dimeric proteins degrade at a rate much slower than all other reactions of the system, which is typically the case in E.coli [37]. Here, the goal is to expound the effect of $N_{p}$ and $N_{d}$ on the various properties that characterize the dynamics of the autoregulatory motif. Previous studies have separately studied the impact of decoy binding [32] and CNV [30] of an autoregulatory motif on its input-output relationship. Hence this model serves as a good starting point for analysis of more complex regulatory architectures. Using principle of mass action kinetics, the model can be described by a set of coupled ordinary differential equations (ODEs) characterizing the time evolution of average concentrations of relevant chemical species in the system [see Fig. 1(b)]. The number of gene copies and decoys entering the description via the concentration of promoter and decoys, $N_{p}$ and $N_{d}$, are proportional to the total concentration of promoter sites $d$ (where $d_{o}$ and $d_{u}$ stand for concentrations of occupied and unoccupied promoter sites, respectively) and decoy sites $\bar{d}\left(\bar{d}_{o}\right.$ and $\bar{d}_{u}$ are concentrations of occupied and unoccupied decoy sites), where $N_{p}=d / C$ and $N_{d}=\bar{d} / C ; C=10^{9} M$ is the molar concentration of a single molecule in the volume of an $E$ coli cell, as reported earlier [30,37]. To solve these coupled ODEs, we make the simplifying assumption that the binding/unbinding of protein molecules to promoters and decoy sites equilibrates on a timescale which is comparatively faster than that of protein turnover. This assumption is consistent with numerous experimental [37] and theoretical studies [30,41]. Following Bennett et al. [41], we use a quasi-steady-state approximation which exploits this separation of timescales in the system to solve for the transient and steady-state protein concentrations [see Fig. 1(c)]. In particular, we keep track of the total monomer concentration. Details of the calculations can be found in Appendix A. This analytical procedure serves as a recipe for exploring the other motifs in the ensuing sections.

Intriguingly, our model predicts that the mean expression level remains the same for a varying number of decoy sites. However, the time to reach the steady state is enhanced as we increase the number of decoy sites [see Fig. 2(a)]. As the number of decoy sites is enhanced, the possible number of configurations in the system increases, resulting in a higher amount of time to reach steady-state levels. This result is further confirmed by considering the time it takes to reach half the level of steady-state protein expression. This time is called the half-times. We find that in the absence of decoys, these half-times increase linearly with copy number $N_{p}$ [see Fig. 7(a), in Appendix]. Moreover, half-times increase 
(a)

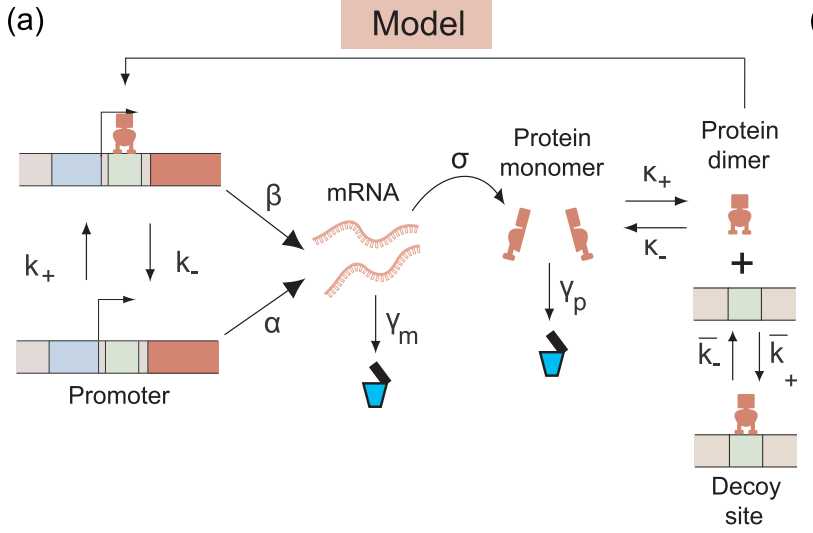

(b) Model reduction using Quasi steady-state approximations

Fast processes:

Promoter/decoy-protein dimer binding/unbinding

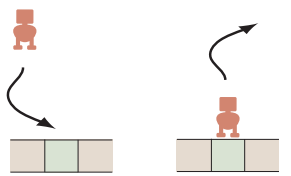

Dimerization/de-dimerization
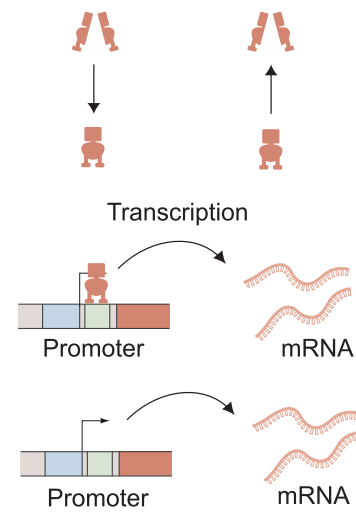

Slow processes: Translation

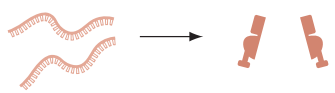

FIG. 1. (a) Schematic representation of various processes for a positive autoregulatory motif in the presence of decoy sites. The promoter switches between a protein bound and empty states with rates $k_{-}$and $k_{+}$, respectively. Basal and regulated mRNA production rates are $\alpha$ and $\beta$. The mRNA translation rate is $\sigma$, which leads to monomeric protein production. Monomers reversibly bind to form a dimer with rate $\kappa_{+}$, which subsequently can dissociate into monomers at rate $\kappa_{-}$. mRNA and monomeric proteins degrade at rates $\gamma_{m}$ and $\gamma_{p}$, respectively. We assume that the dimeric protein can bind or unbind to the promoter or decoys with the same rates. (b) Governing equations characterizing the time evolution of the relevant variables in the system. Variables refer to concentrations of protein monomers $x$, protein dimers $y$, unoccupied and occupied promoters $d_{u}$ and $d_{o}$, unoccupied and occupied decoys $\bar{d}_{u}$ and $\bar{d}_{o}$, and mRNA $m$. (c) Separation of timescales, list of fast and slow processes we consider (mRNA and protein degradation rates are not shown).

monotonically as a function decoy number and exhibit rapid increases for higher numbers of decoys. For smaller decoy numbers, half-times do not change in an appreciable manner, while for higher decoy numbers the change in half-times is significant. The point of rapid increase shifts to higher values of decoys as the gene copy number increases [see Fig. 2(b)]. In other words, there exists a "tug-of-war" between gene copies and decoys which dictates the transient dynamics of autoregulation. It must be noted that the above-mentioned findings are a result of the prefactor method we employ. These results hold when we solve for the full system of equations, as shown in Figs. 2(a) and 2(b), for a wide range of parameter values (see Fig. 7, in Appendix).

\section{B. Toggle switch}

The toggle switch has attracted a lot of attention in the recent past, since it is one of the simplest regulatory motifs that exhibit bistability [38-40,42,43]. This switch is often posited as the canonical model to characterize how multicellular organisms make "either/or-like" cell-fate decisions during development. Other instances of bistability include the lysis-lysogeny circuit of bacteriophage lambda [44], the lac operon repressor system in E. coli $[45,46]$, and cellular signal transduction pathways [47-50].

A toggle switch consists of two genes that mutually repress each other, as shown in Fig. 3(a). This system exhibits two stable equilibrium points (also known as attractors) in which one of the two genes is expressed at a high level while the other is expressed at a low level. In addition to these states, there is one unstable equilibrium point at which both the genes are expressed at some intermediate level. Associated with the two attractors are distinct regions of gene expression space of the two genes that are referred to as basins of attraction; a system evolved from an initial point within a basin of attraction eventually iterates into the corresponding attractor [see 
(a)

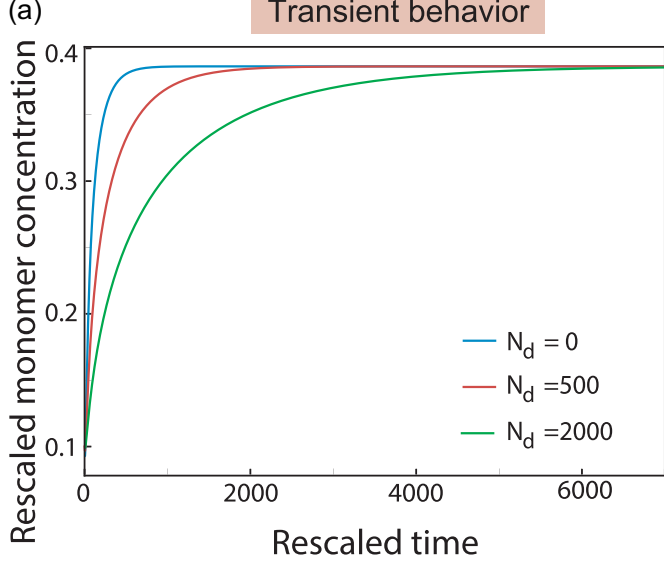

(b)

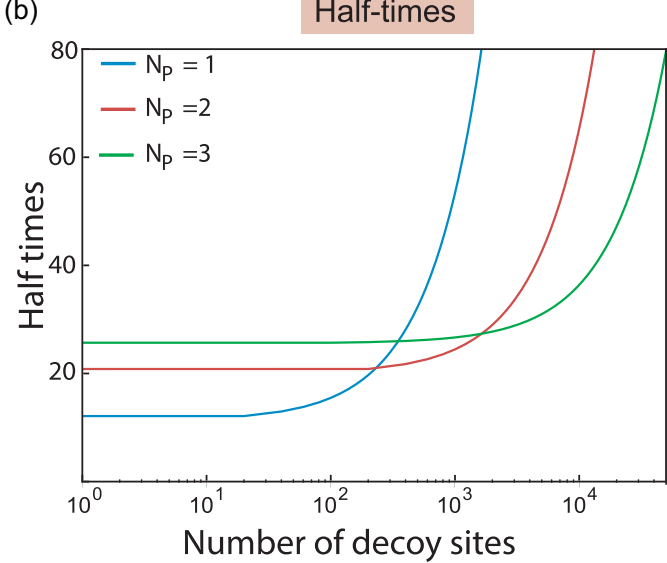

FIG. 2. (a) Attainment of steady state of total monomer concentration. The presence of decoys affects the transient dynamics; the time it takes to reach the steady state is enhanced as the number of decoys in increased. (b) Half-times as functions of decoy copy numbers are shown for different copy numbers of the motif. Here half-times is defined as the time it takes to reach half of the steady-state monomer concentration.

Fig. 3(b)]. A straight line called the separatrix delimits the two basins of attraction. Our aim in this paper is to investigate whether the presence of CNV and decoy binding affect these dynamical features, particularly the basins of attraction of a toggle switch. In order to achieve this goal, we assume that in addition to $N_{p}$ copies of the switch, there exist two sets

(a)

\section{Model}

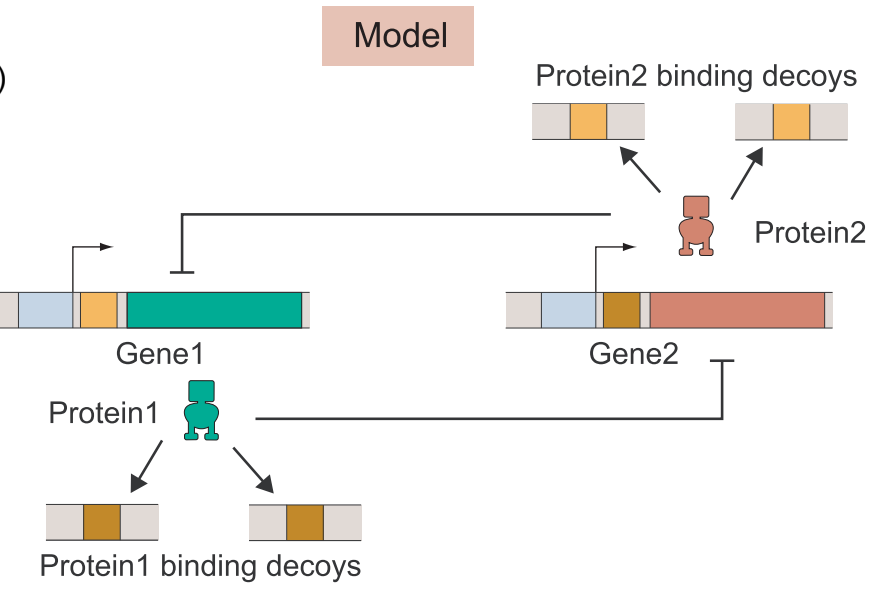

\section{Homogeneous decoys $\mathrm{N}_{\mathrm{d} 1}=\mathrm{N}_{\mathrm{d} 2} ; \mathrm{N}_{\mathrm{p}}=1$}

(b)

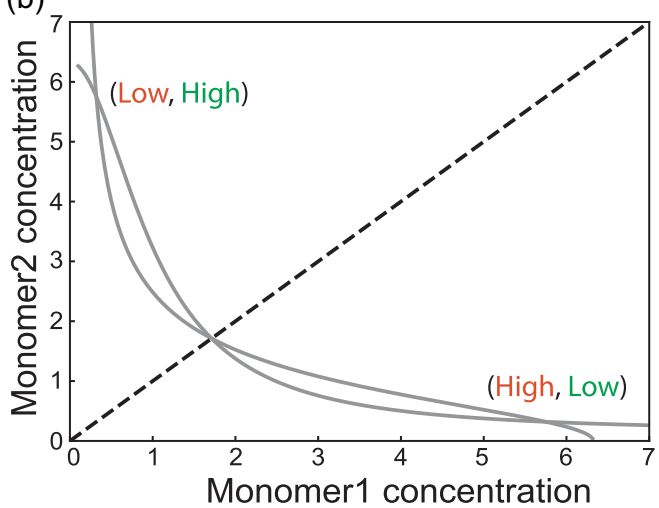

(c)

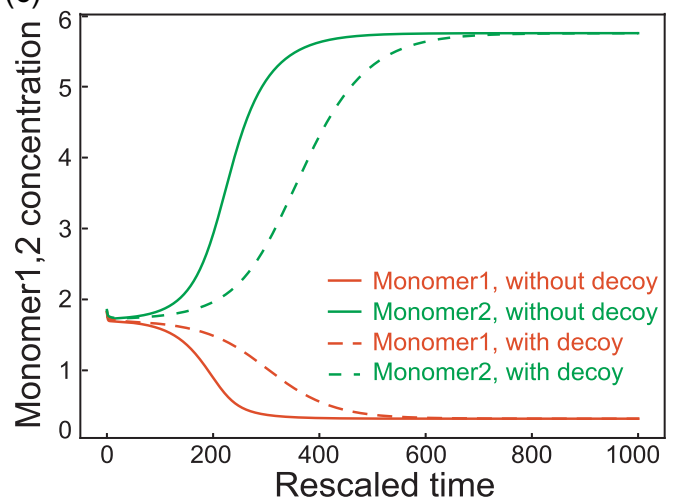

FIG. 3. (a) Cartoon of the toggle switch. Two genes repress each other. The protein products pertaining to the genes can bind to decoys. (b) Phase space of the toggle switch in the presence of homogeneous decoys. The dashed line indicates a separatrix which defines the two stable states of the toggle switch (high, low) and (low, high). The gray lines are nullclines for the toggle switch system which intersect at two stable and one unstable fixed points (see Appendix B for details). (c) An example of the dynamics in the basin of attraction for (low, high) state with and without decoys. Note that the presence of decoys increases the time to reach the steady state. 

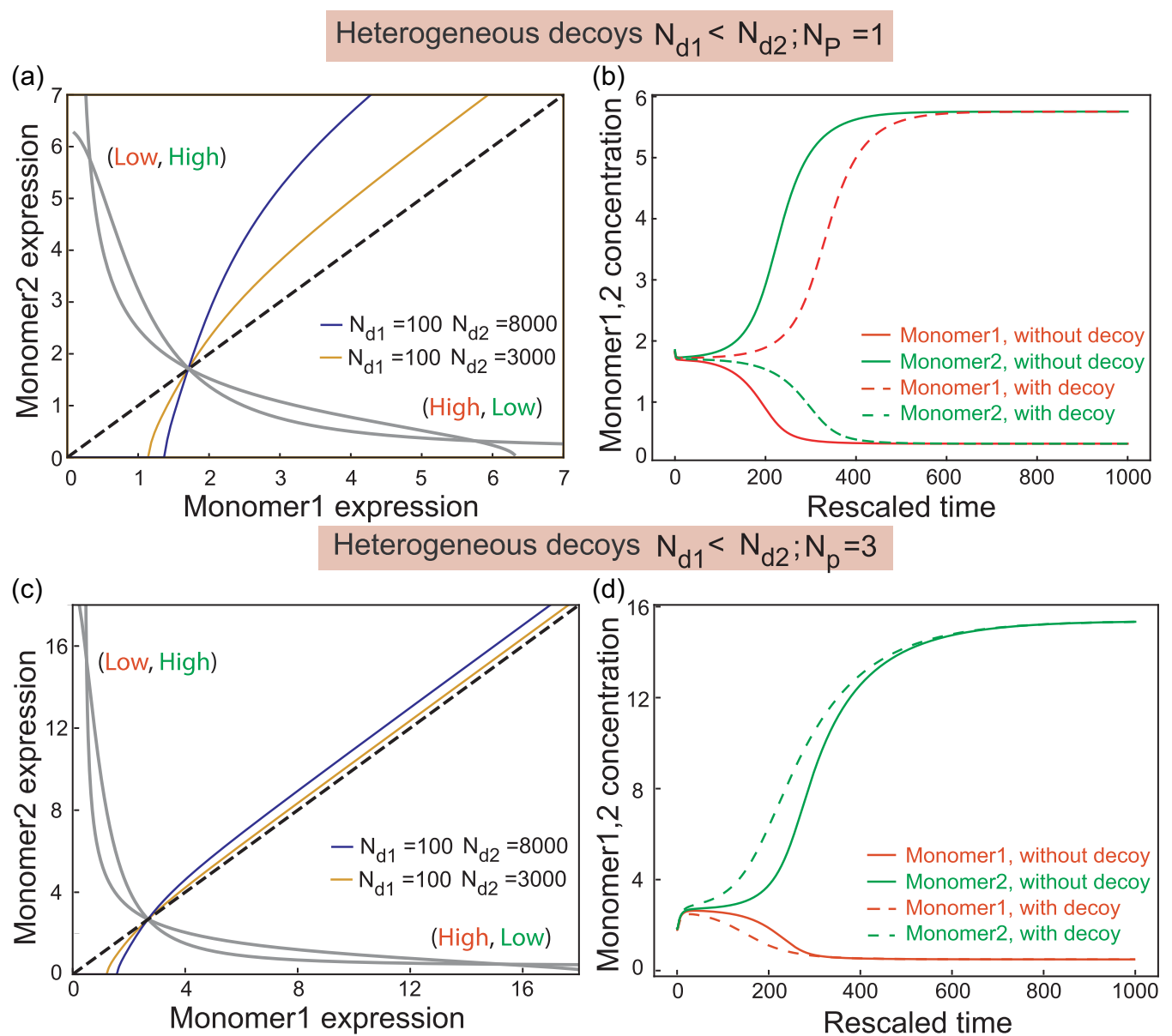

FIG. 4. (a) Phase space of the toggle switch in the presence of heterogeneous decoys, i.e., when the numbers of decoys corresponding to two genes are different (number of decoys for protein 2 is higher than the number for protein 1). The separatrix begins to exhibit a curvature that changes with the degree of dissimilarity in decoy numbers. The basin of attraction associated with (high, low) state thus expands at the expense of the one related to (low, high) state. The gray lines are nullclines for the toggle switch system, which intersect at two stable and one unstable fixed points (see the Appendixes for details). (b) The example of Fig. 2(b) is replotted in the presence of dissimilar decoys. Initial conditions that evolved to (low, high) state earlier evolved to (high, ow) state. (c) Phase space of the toggle switch for $N_{p}=3$ copies of the switch in the presence of heterogeneous decoy binding. Increase in gene copy number rescues the effect of heterogeneous decoy binding; the effect of decoys is suppressed. It must be noted that the average expression level of both genes is higher when the copy number of the switch is higher. (d) Such a rescue effect is further evident by the transient behavior of the system; time to reach the steady state is reduced.

of decoy sites ( $N_{d 1}, N_{d 2}$ copies, respectively) that the protein products of the two genes can bind to. The analysis is carried out by employing the same mathematical formalism as before (see Appendix B for details).

First we consider the scenario when the copy number of switch is 1 and the two species of decoys are homogeneous, i.e., the number of both sets of decoy sites is equal with identical protein binding affinities. Under these conditions, the steady-state behavior of the toggle switch is not altered; the attractors and the corresponding basins of attraction remain identical to the usual toggle switch. However, the transient dynamics are impacted as in the autoregulatory motif in the presence of decoys. The time to reach the steady state is slowed down substantially [see Fig. 3(c)] due to the system having to sample a larger space of possible microstates.

Next we consider the scenario in which two species of decoys are heterogeneous; the number of decoys pertaining to the protein product of gene 2 is higher than that of gene 1 .
However, their protein binding affinities are equal. As shown in Fig. 4(a), the basin of attraction of gene 2 is substantially decreased at the expense of that of gene 1 . Let us consider the case in which in the absence of decoys, gene 2 expression is high and the expression of gene 1 is low. Now, introducing a higher number of decoys corresponding to gene 2 leads to its protein product on average getting occupied more often than that of gene 1 . This results in a reduction in the average level of repression gene 2 exerts on gene 1 . Therefore gene 1 can switch to a higher level of expression and in turn, represses gene 2. Thus an initial point in the expression space of the two genes that previously evolved to the first attractor now go to the second one, as shown in Fig. 4(b). This effect remains intact when the two sets of decoys are identical in numbers but bind to the respective proteins with unequal affinities [see Fig. 7(b), in Appendix].

A key result of our paper is that the effect of heterogeneous decoy binding is significantly suppressed when the copy number of the toggle switch is enhanced. As shown in Fig. 4(c), 

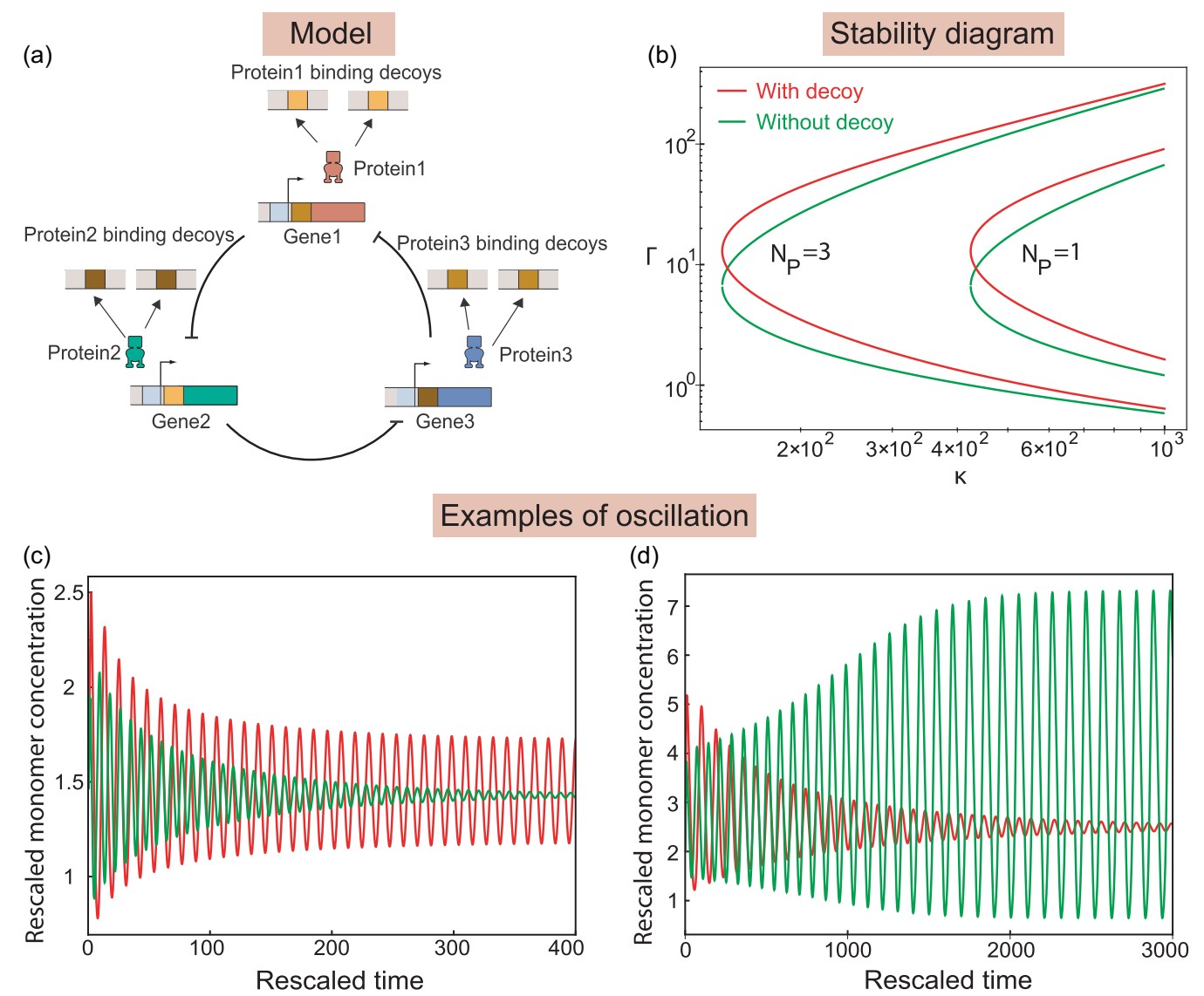

FIG. 5. (a) Cartoon of the repressilator. Three genes repress each other in a cyclic structure. The proteins produced from these genes bind to decoy sites. (b) Stability diagram in $\Gamma-\kappa$ parameter space, where $\Gamma=\gamma_{p} / \gamma_{m}$ and $\kappa=\alpha \sigma /\left(\gamma_{m} \gamma_{p}\right)$ [see Fig. 1(c) and Appendixes for details of the parameters]. The blue curve is for the case without decoys, while the red curve indicates the case when decoys are added. (c) We show stability diagrams for $N_{p}=1,3$ with decoy number $N_{d}=1000$. A part of the steady-state regime becomes oscillatory in the presence of decoys and vice versa. (d) An example of dynamics from the region exhibiting oscillatory behavior without decoys becomes steady in the presence of decoys. (e) A complementary example from the region for the opposite case is shown.

the basins of attraction can be rescued at high copy number. Consequently, points in the basin of attraction of one attractor that switched to the other due to heterogeneous decoys returns to the former attractor when the copy number is increased [see Fig. 4(d)]. As such, our theory makes specific predictions about how an interplay between $\mathrm{CNV}$ and decoy binding alters the dynamical properties of the toggle switch.

\section{Repressilator}

The repressilator typically consists of three genes coupled in a cyclic structure, such that each gene represses the next [see Fig. 5(a)], generating oscillations in protein concentrations $[7,10]$. There exist numerous examples of naturally occurring repressilators [51]. A classic example is that of the circadian clock, which oscillates according to the day-night cycle [52,53]. In mammals a set of three genes, cryptochrome (Cry), period (Per), and Rev-erb, serve as a major core element of the circadian network [54]. A repressilator is responsible for controlling circadian timing in A. thaliana. Synthetic circuits built from well-characterized genetic parts can also exhibit oscillations [55,56].
As a dynamical system, the repressilator exhibits stable limit cycles appearing due to a supercritical Hopf bifurcation of the stable equilibrium point [30,41]. Consequently, the system exhibits oscillations for a wide range of parameters. In other words, the protein products of each of the three genes in the circuit oscillate in time. The corresponding bifurcation diagram displays a separatrix demarcating the oscillatory from the steady-state regime. We are interested in unraveling the consequences of $\mathrm{CNV}$ and decoy binding of proteins expressed from these genes upon the dynamical behavior of repressilator.

To carry out such an analysis, we consider the number and protein binding affinity of the three sets of decoy sites corresponding to three kinds of protein products in the system to be identical. Numerical simulations show that the copy number of the motif and decoys significantly alters the oscillatory regime. In particular, our analysis evinces that a considerable region in the parameter space that exhibited steady-state behavior turns oscillatory in the presence of CNV and decoy sites, as evident from the standard bifurcation diagram [see Fig. 5(b)]. In addition, there emerges a parameter regime which is no longer oscillatory [for example, see Figs. 5(c) and 5(d)]. 

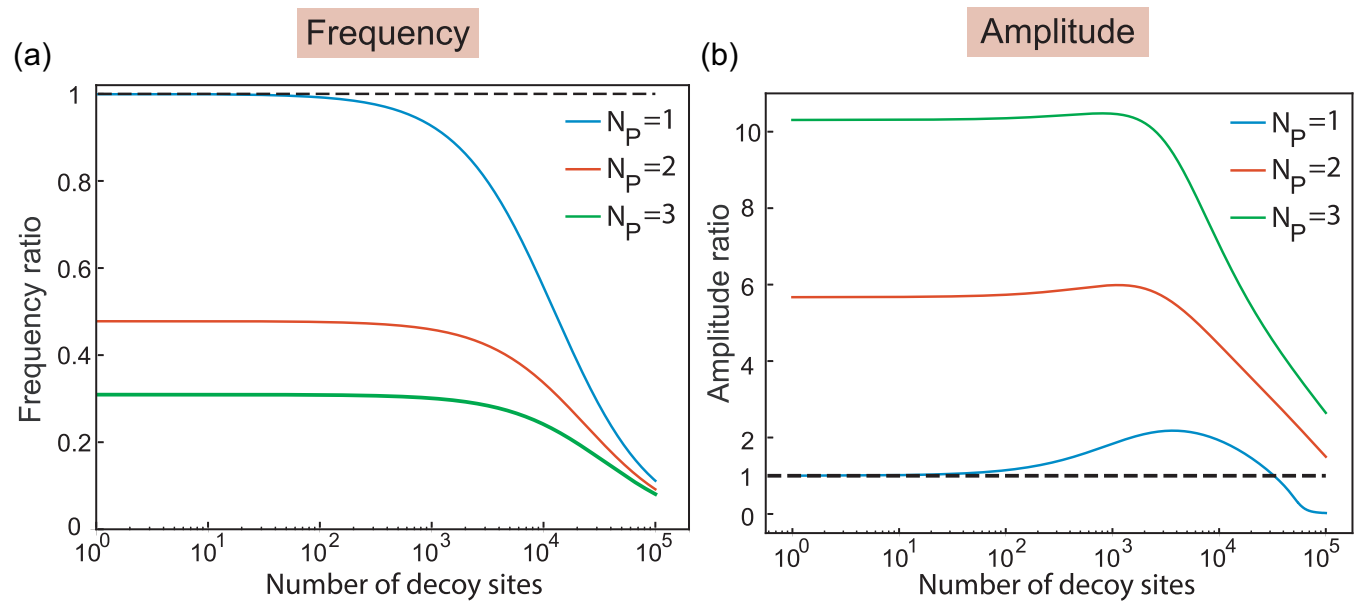

FIG. 6. (a) Frequency ratio of oscillation with and without decoy binding is shown. Ratios for three different gene copy numbers are shown. The dashed line in black indicates $\omega_{r}=1$ for reference. (b) Amplitude ratios with and without decoys for different copy numbers of genes are shown. It shows a nonlinear behavior as a function of decoy number; initially it does not vary much but then shows a hump followed by rapid decline as the decoy number is increased. The dashed line in black indicates $A_{r}=1$ for reference.

We further study the impact of CNV and decoy binding on the frequency (also known as Hopf frequency) and amplitude of oscillation. An expression of frequency $\omega$ has been derived analytically which involves the gene copy number $N_{p}$ and decoy number $N_{d}$ (see Appendix C for details). We seek to explore how this frequency changes as a function of $N_{p}$ and $N_{d}$. In particular, the frequency ratio $\omega_{r}$ is defined as the ratio of $\omega$ and $\omega_{0}$, which is the frequency for $N_{p}=1$ and $N_{d}=0$. The frequency ratio $\omega_{r}$ monotonically decreases with increasing decoy number. While the overall frequency is further reduced as the gene copy number is increased, the rate of decrease in frequency ratio is smaller for higher gene copy numbers, as shown in Fig. 6(a). Once again, the influence of decoys is seen to be negated by CNV.

Interestingly, we discover that the amplitude of oscillation demonstrates both an increase and decrease in the presence of decoy binding sites. We define an amplitude ratio $A_{r}$ (similar to $\omega_{r}$ ). The ratio is stable for small $N_{p}$ and $N_{d}$. A hump in the curve appears for moderate $N_{d}$ for $N_{p}=1$, which indicates the region of high amplitude followed by a rapid decline for high $N_{d}$. With higher gene copy numbers, the hump begins to disappear and the subsequent fall in the ratio is relatively sharper, as shown in Fig. 6(b). This behavior can be further confirmed by observing the monomer concentration as a function of the number of decoy sites, as shown in Fig. 10 (see Appendix). It must be noted that for a large $N_{d}$, the repressilator moves from the oscillatory to the steady-state regime. This behavior is sensitive to various parameters of the motif, such as monomer degradation rate, protein binding affinity of decoys, etc.

\section{DISCUSSION}

In this manuscript we have investigated how gene copy number variation and presence of decoy sites together modulate the dynamical properties of three well-known regulatory motifs. Copy number variation of genes and addition of decoy sites invoke a competition between the promoters and decoys for the pool of regulatory proteins in the system $[30,33,34]$. Such resource sharing couples the dynamics of genetic circuits with cell physiology in general and provides a rich context in which these circuits operate in cells [57]. This is particularly pertinent in engineering synthetic circuits, which entails a firm quantitative understanding of how the various contexts in a cell impacts the circuit behavior $[35,58]$. Recent studies in the field of regulatory biology have started exploring the impact of resource sharing on the dynamics of genetic circuits [30] and single genes $[5,12,18,31-35,59,60]$. However, a comprehensive theoretical understanding is still lacking. In this manuscript we use the prefactor method, as introduced earlier [41], to study the transient and steady-state properties of the motifs under investigation (please see Table I).

Our study reveals that for the toggle switch, the basins of attraction of the two attractors can be significantly altered in the face of decoy binding. Similar results were obtained previously, when Lyons et al. [12] showed that the addition of a downstream component or load to the toggle switch alters the underlying potential energy landscape. The downstream component in that case could be a protein or a small molecule such that the bound complex prevents one kind of repressor from binding to and repressing its conjugate promoter. In effect, our system invoked the same effect of altering the effective concentration of one of the proteins by occupying them with more decoy sites. Intriguingly, we find that a relatively smaller increase in gene copy number can dramatically suppress this effect. This result implies that using multiple copies of synthetic circuits in a cell can keep the dynamics of these circuits relatively insulated. Moreover, the findings in this paper may have important biological implications. For instance, the toggle switch is often invoked as a minimal model of cellular decision-making during mammalian development, where cells need to choose between alternate fates. Often cell types associated with these fates need to be produced in different proportions. Our results suggest an exciting possibility of transcription factor re- 
TABLE I. Summary of observations for the three motifs with change in promoter and decoy copy numbers.

\begin{tabular}{ll}
\hline \hline Motif & Observations \\
\hline Autoregulator & $\begin{array}{c}\text { Change in promoter concentration influences the steady-state levels of the gene. } \\
\text { Increase in decoy concentration increases half-times to attain the steady-state level. } \\
\text { Toggle switch } \\
\text { Anmetry in binding affinities to decoy sites alters the basins of attraction corresponding to the stable states. } \\
\text { Increase in promoter concentration counterbalances the effects introduced by the increase in decoy sites. } \\
\text { Increase in promoter concentration expands the oscillatory regime in the stability diagram. } \\
\text { Change in decoy concentration leads to emergence of a new oscillatory regime while frequency and amplitude } \\
\text { are affected in a nonlinear fashion. }\end{array}$ \\
\hline
\end{tabular}

source sharing being a potential mechanism for controlling the proportion of cells belonging to different cell types during development.

Introduction of decoy sites impact the oscillatory behavior of a repressilator in multiple ways. The stability diagram is altered significantly as CNV and decoy binding are employed. As such, both these features increase the parameter space of oscillations. Amplitude and frequency of oscillations can be widely tuned as a function of the number of decoy sites and gene copy number. The impacts of decoy binding are consistent with a previous study that explored the impact of decoy binding on NF-kB networks which exhibit oscillation [18]. Although the concomitant circuit architecture was different, they observed an alteration in amplitude and period of oscillations. On the one hand, our results provide constraints on how to build a synthetic repressilator, and on the other, the results imply enhanced tunability of the circuit's dynamical behavior. Whether such differential control is functionally utilized by cells is an intriguing question.

It must be emphasized that the prefactor method we employ to solve for the transient and steady-state properties of the system suffers from notable limitations. This method is a quasi-steady approximation with an appropriate correction factor that appears due to the correct identification of the truly slow variable in the system. Such a reduction scheme heavily relies upon the fact that timescale separation exists in the system. If different chemical species of the system react with similar timescales, i.e., are not separable into sets of "slow" and "fast" reactions, then this reduction method is not applicable and our results would not hold. Moreover, the method ignores the stochastic fluctuations introduced due to the intrinsic randomness of biochemical reactions present in the system [41].

Overall, our study evinces that $\mathrm{CNV}$ and decoy binding are in a tug-of-war in terms of governing the behavioral properties of genetic motifs. Changes in gene copy number and decoy number can potentially function as tuning knobs within a nonlinear dynamical system as another layer of regulation.

All the codes for the computational analysis and generating the subsequent plots are done in Python and can be found on Github [61].

\section{ACKNOWLEDGMENT}

S.D. is grateful to Arnd Bäcker for his generous support during this work.

\section{APPENDIX A: AUTOREGULATION}

We begin with the autoregulatory motif-the protein product of a single gene regulates its own expression. Consider a simplified setting wherein protein monomers dimerize to create dimers which can bind or unbind with available promoters and decoys. Promoter bound protein dimers lead to transcription, while decoy bound dimers remain inert. A detailed description of the model is given in the main text in Fig. 1(a).

The dynamics of the system is captured by the following set of coupled differential equations:

$$
\begin{aligned}
\dot{x} & =2 \kappa_{-} y-2 \kappa_{+} x^{2}+\sigma m-\gamma_{p} x \\
\dot{y} & =\kappa_{+} x^{2}-\kappa_{-} y+k_{-} d_{o}+\bar{k}_{-} \bar{d}_{o}-k_{+} d_{u} y-\bar{k}_{+} \bar{d}_{u} y \\
\dot{d}_{o} & =k_{+} d_{u} y-k_{-} d_{o} \\
\dot{d}_{u} & =-k_{+} d_{u} y+k_{-} d_{o} \\
\dot{\bar{d}}_{o} & =\bar{k}_{+} \bar{d}_{u} y-\bar{k}_{-} \bar{d}_{o} \\
\dot{\bar{d}}_{u} & =-\bar{k}_{+} \bar{d}_{u} y+\bar{k}_{-} \bar{d}_{o} \\
\dot{m} & =\alpha d_{u}-\gamma_{m} m+\beta d_{o} .
\end{aligned}
$$

These equations represent the time evolution of the average concentration of following seven species:

$x \equiv$ protein monomers,

$y \equiv$ protein dimers,

$m \equiv$ mRNA,

$d_{u} \equiv$ unoccupied promoter sites,

$d_{o} \equiv$ occupied promotor sites,

$\bar{d}_{u} \equiv$ unoccupied decoy sites,

$\bar{d}_{o} \equiv$ occupied decoy sites.

The parameters involved are listed below:

$\sigma \equiv$ translation rate,

$\alpha \equiv$ basal transcription rate,

$\beta \equiv$ regulated transcription rate,

$\gamma_{m} \equiv$ degradation rate of mRNA,

$\gamma_{p} \equiv$ degradation rate of protein monomers,

$k \pm \equiv$ binding and dissociation rates of the protein to a promoter,

$\bar{k} \pm \equiv$ binding and dissociation rates of the protein to a decoy site,

$\kappa \pm \equiv$ binding and dissociation rates of the protein to themselves. 
We have the constraint that the total amount of promoters and decoys remains conserved:

$$
\begin{aligned}
& d_{u}+d_{o}=d, \\
& \bar{d}_{o}+\bar{d}_{u}=\bar{d},
\end{aligned}
$$

where $d$ and $\bar{d}$ are total concentrations of promoter sites and of decoy sites, respectively. We substitute these expressions into Eq. (A1) and use the recalling that $d=N_{p} C$ and $\bar{d}=N_{d} C$. Here $N_{p}$ and $N_{d}$ are the promoter and decoy copy numbers, respectively. $C=10^{-9} \mathrm{M}$ is the conversion factor denoting the concentration of a single molecule in the volume of an E. coli cell [30,37]. Moreover, the degradation of dimers is ignored here for analytical tractability.

\section{Multiple timescale analysis}

The governing Eqs. (A1) is a set of seven dimensional nonlinear coupled differential equations. But as described in several previous studies [30,37], the system exhibits multiple timescales that may be used to reduce the effective dimensions of the system, which leads to simplification of the subsequent mathematical and computational analysis.

We assume that the dimerization and regulatory binding processes are fast compared to transcription, translation, and degradation. These assumptions hold for a large number of bacterial and viral genes [30,37]. Therefore Eqs. A1(b)-A1(f) involve fast processes, while Eqs. A1(a) and A1(g) are slow. Consequently, the fast reactions will reach their steady states earlier compared to their slow counterparts. By setting derivatives of $y, d_{u}, d_{o}, \bar{d}_{u}$, and $\bar{d}_{o}$ to zero, we obtain

$$
\begin{aligned}
& 0=\kappa_{+} x^{2}-\kappa_{-} y+k_{-} d_{o}+\bar{k}_{-} \bar{d}_{o}-k_{+} d_{u} y-\bar{k}_{+} \bar{d}_{u} y \\
& 0=k_{+} d_{u} y-k_{-} d_{o} \\
& 0=-k_{+} d_{u} y+k_{-} d_{o} \\
& 0=\bar{k}_{+} \bar{d}_{u} y-\bar{k}_{-} \bar{d}_{o} \\
& 0=-\bar{k}_{+} \bar{d}_{u} y+\bar{k}_{-} \bar{d}_{o} .
\end{aligned}
$$

Thus we obtain the steady-state values as below:

$$
\begin{gathered}
y=c_{p} x^{2} \\
d_{u}=\frac{C N_{p}}{1+c_{p} c_{d} x^{2}} \\
d_{o}=\frac{C N_{p} c_{p} c_{d} x^{2}}{1+c_{p} c_{d} x^{2}} \\
\bar{d}_{u}=\frac{C N_{d}}{1+c_{p} \bar{c}_{d} x^{2}} \\
\bar{d}_{o}=\frac{C N_{d} c_{p} \bar{c}_{d} x^{2}}{1+c_{p} \bar{c}_{d} x^{2}},
\end{gathered}
$$

where, $c_{p}=\frac{\kappa_{+}}{\kappa_{-}}, c_{d}=\frac{k_{+}}{k_{-}}$, and $\bar{c}_{d}=\frac{\bar{k}_{+}}{\bar{k}_{-}}$.

These steady-state values can now be substituted in Eqs. A1(a) and A1(g) to obtain

$$
\begin{aligned}
\dot{x} & =\sigma m-\gamma_{p} x \\
\dot{m} & =\frac{N_{p} c_{p} c_{d} x^{2}}{1+c_{p} c_{d} x^{2}}\left[\alpha+\beta c_{p} c_{d} x^{2}\right]-\gamma_{m} m .
\end{aligned}
$$

The continuous approximation scheme described above is called the quasi-steady-state approximation (QSSA) [30,37,41] and is popular among theorists to model the dynamics of the network motif. However, in order to track the transients correctly, an important correction in the form of a prefactor was proposed [41]. We find that the prefactor method naturally allows for inclusion of copy number variation and presence of decoys, as described below.

\section{The prefactor method}

We note that reaction $\dot{x}$ is actually a mixture of slow (translation and degradation) and fast (dimerization and disassociation) reactions. Therefore a true slow variable is the total concentration of protein molecules (in any form):

$$
n_{x}=x+2 y+2 d_{o}+2 \bar{d}_{o} .
$$

Time evolution of $n_{x}$ is given by

$$
\dot{n}_{x}=\sigma m-\gamma_{p} x
$$

Now, substituting steady values in Eq. (A10),

$$
n_{x} \approx x+2 c_{p} x^{2}+2 C N_{p} \frac{c_{p} c_{d} x^{2}}{1+c_{p} c_{d} x^{2}}+2 C N_{d} \frac{c_{p} \bar{c}_{d} x^{2}}{1+c_{p} \bar{c}_{d} x^{2}}
$$

We can now express $\dot{n}_{x}$ as follows:

$$
\dot{n}_{x}=\dot{x} \frac{\partial n_{x}}{\partial x}=\dot{x} p(x),
$$

where the prefactor $p(x)$ is given by

$$
\begin{aligned}
p(x)= & 1+4 c_{p} x+4 C N_{p} \frac{c_{p} c_{d} x}{\left(1+c_{p} c_{d} x^{2}\right)^{2}} \\
& +4 C N_{d} \frac{c_{p} \bar{c}_{d} x}{\left(1+c_{p} \bar{c}_{d} x^{2}\right)^{2}} .
\end{aligned}
$$

A new system of equations for time evolution of $x$ and $m$ is obtained using Eqs. (A11) and (A14):

$$
\begin{aligned}
p(x) \dot{x} & =\sigma m-\gamma_{p} x \\
\dot{m} & =\frac{C N_{p} c_{p} c_{d} x^{2}}{1+c_{p} c_{d} x^{2}}\left[\alpha+\beta c_{p} c_{d} x^{2}\right]-\gamma_{m} m .
\end{aligned}
$$

Note that any term involving decoy sites does not appear explicitly in the Eq. (A15). Their influence is buried in the prefactor $p(x)$ [see Eq. (A14)].

\section{Comparison of the prefactor method with the full system}

On comparing the results obtained from the prefactor method and those from the full system (without any reduction scheme used), we find that

(1) Both the methods lead to qualitatively similar dynamics.

(2) Increasing decoy sites results in longer transients in the full system. This behavior once again is captured by the prefactor method.

The corresponding plots are given in Fig. 7. 

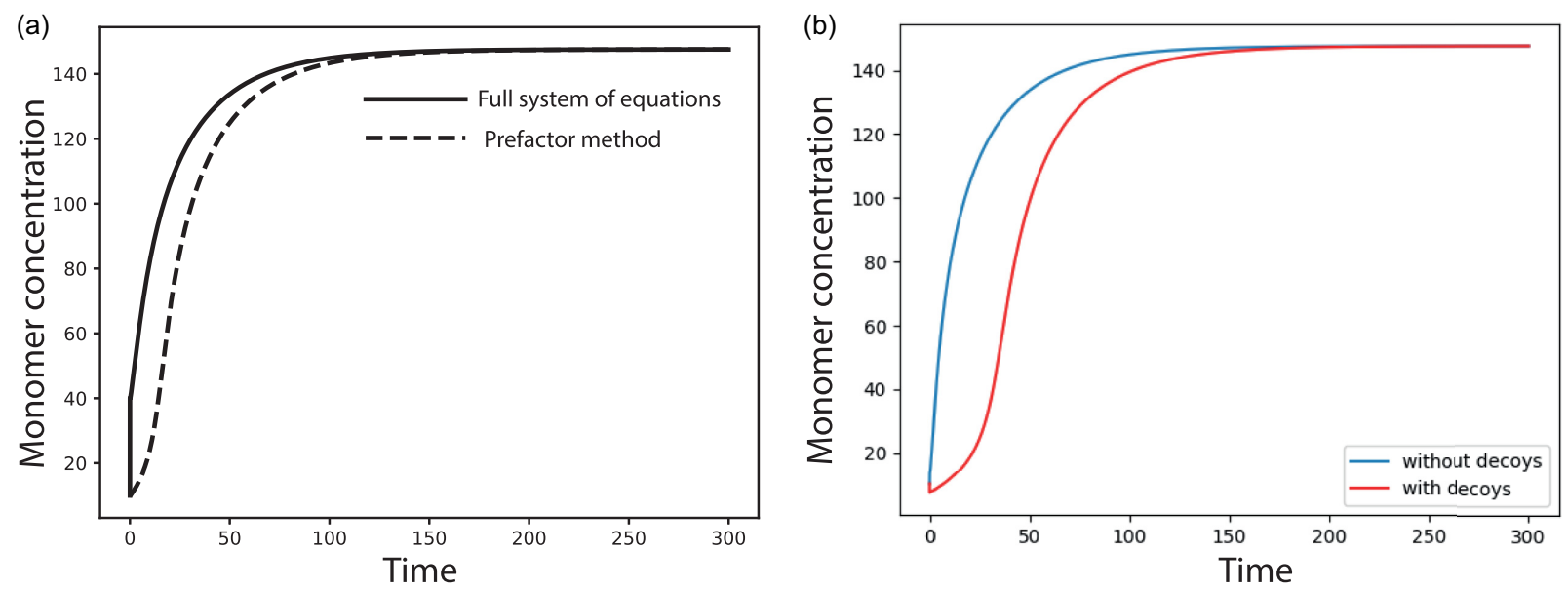

FIG. 7. (a) Comparison of the prefactor method (dashed curve) with the full unreduced system (solid curves). (b) Transient dynamics due to increasing decoy sites is sustained for longer times in the full system. The blue curve is for the case with decoy sites, and the red curve shows the case with decoy sites. This behavior is again similar to what we have observed with the prefactor method.

\section{Computation of half-life $T_{1 / 2}$}

We define $T_{1 / 2}$ as the time the system takes from an initial concentration of proteins (and mRNA) to reach one-half of its steady-state value. Figure 2 in the main text shows the effect of decoys on $T_{1 / 2}$ when $N_{p}=1$.

To determine a reliable value of $T_{1 / 2}$, we consider an ensemble of 100 random initial conditions drawn from a uniform distribution in a neighborhood $\Delta \approx 10^{-2}$ of the stable equilibrium point. For a given $N_{p}, T_{1 / 2}$ averaged over the ensemble is then taken to be the value of half-life. In the absence of decoys, the computed values for various $N_{p} \in\{1,2,3,4,5,6\}$ are plotted in Fig. 8(a). A similar procedure is used to compute $T_{1 / 2}$ in the presence of decoy sites shown in Fig. 2 in the main text.

\section{Parameters}

We use the following parameter values to perform computations [30,37,41]:

$C=10^{-9} \mathrm{M}, \quad c_{p}=10^{7} \mathrm{M}^{-1}, \quad c_{d}=10^{7} \mathrm{M}^{-1}, \quad \bar{c}_{d}=$ $10^{7} \mathrm{M}^{-1}, \sigma=0.5$.

Time evolution of monomer concentration and half-time computations of Figs. 2(a) and 2(b) were done using the following model parameters:

$\alpha=0.025 \mathrm{~min}^{-1}$

$\beta=1.3 \mathrm{~min}^{-1}$,

$\gamma_{p}=0.05 \mathrm{~min}^{-1}, \gamma_{m}=$ $0.10 \mathrm{~min}^{-1}[30,37,41]$.

\section{APPENDIX B: TOGGLE SWITCH}

A toggle switch motif contains two genes (see the full set of reactions in Fig. 9) with two possible states of expression(low, high) and (high, low)—which are obtained by one gene partially inhibiting transcription of the other. We assume regulated transcription rates $\beta_{1,2}$ to be smaller than basal transcription rates $\alpha_{1,2}$, i.e., $\beta_{1,2}<\alpha_{1,2}$. For simplicity, we keep $\beta_{1}=\beta_{2}(=\beta), \alpha_{1}=\alpha_{2}(=\alpha)$, and $\gamma_{p, 1}=\gamma_{p, 2}\left(=\gamma_{p}\right)$.
We use Eqs. (A15) and the prefactor [see Eq. (A14)] to write the model for the dynamics of the toggle switch as follows:

$$
\begin{aligned}
p\left(x_{i}\right) \dot{x}_{i} & =\sigma m_{i}-\gamma_{p} x_{i}, \\
\dot{m}_{i} & =\frac{\alpha C N_{p}}{1+c_{p} c_{d} x_{j}^{2}}+\frac{\beta C N_{p} c_{p} c_{d} x_{j}^{2}}{1+c_{p} c_{d} x_{j}^{2}}-\gamma_{m} m_{i},
\end{aligned}
$$

where $i \in\{1,2\}, j \in\{2,1\}$.

We use the following rescalings to simplify Eqs. (B1), $\gamma_{m} t \rightarrow t, \sqrt{c_{d} c_{p}} x_{i} \rightarrow x_{i}$, and $\left(\sigma \sqrt{c_{d} c_{p}}\right) /\left(\gamma_{m} \Gamma\right) m_{i} \rightarrow m_{i}$, to obtain

$$
\begin{aligned}
p\left(x_{i}\right) \dot{x}_{i} & =-\Gamma\left(x_{i}-m_{i}\right), \\
\dot{m}_{i} & =\frac{\kappa_{1} \tilde{N}_{p}}{1+x_{j}^{2}}+\frac{\kappa_{2} \tilde{N}_{p} x_{j}^{2}}{1+x_{j}^{2}}-m_{i},
\end{aligned}
$$

where $\Gamma=\gamma_{p} / \gamma_{m}, \kappa_{1}=\alpha \sigma /\left(\gamma_{m} \gamma_{p}\right), \kappa_{2}=\beta \sigma /\left(\gamma_{m} \gamma_{p}\right), \tilde{N}_{p}=$ $\sqrt{c_{d} c_{p}} C N_{p}$, and $i \in\{1,2\}, j \in\{2,1\}$.

The prefactor becomes

$$
p\left(x_{i}\right)=1+4 r x_{i}+\frac{4 \tilde{N}_{p} x_{i}}{\left(1+x_{i}^{2}\right)^{2}}+\frac{4 \tilde{N}_{d} \epsilon x_{i}}{\left(1+\epsilon x_{i}^{2}\right)^{2}},
$$

where $r=\sqrt{c_{p} / c_{d}}$ and $\epsilon=\overline{c_{d}} / c_{d}, \tilde{N}_{d}=\sqrt{c_{d} c_{p}} C N_{d}$. Also, note that the assumption $\beta<\alpha$ translates to $\kappa_{2}<\kappa_{1}$.

\section{Breaking of symmetry of decoys}

Equations (B2) consist of four equations as follows:

$$
\begin{aligned}
p\left(x_{1}\right) \dot{x}_{i} & =-\Gamma\left(x_{1}-m_{1}\right) \\
\dot{m}_{1} & =\frac{\kappa_{1} \tilde{N}_{p}}{1+x_{2}^{2}}+\frac{\kappa_{2} \tilde{N}_{p} x_{2}^{2}}{1+x_{2}^{2}}-m_{1} \\
p\left(x_{2}\right) \dot{x_{2}} & =-\Gamma\left(x_{2}-m_{2}\right) \\
\dot{m}_{2} & =\frac{\kappa_{1} \tilde{N}_{p}}{1+x_{1}^{2}}+\frac{\kappa_{2} \tilde{N}_{p} x_{1}^{2}}{1+x_{1}^{2}}-m_{2} .
\end{aligned}
$$



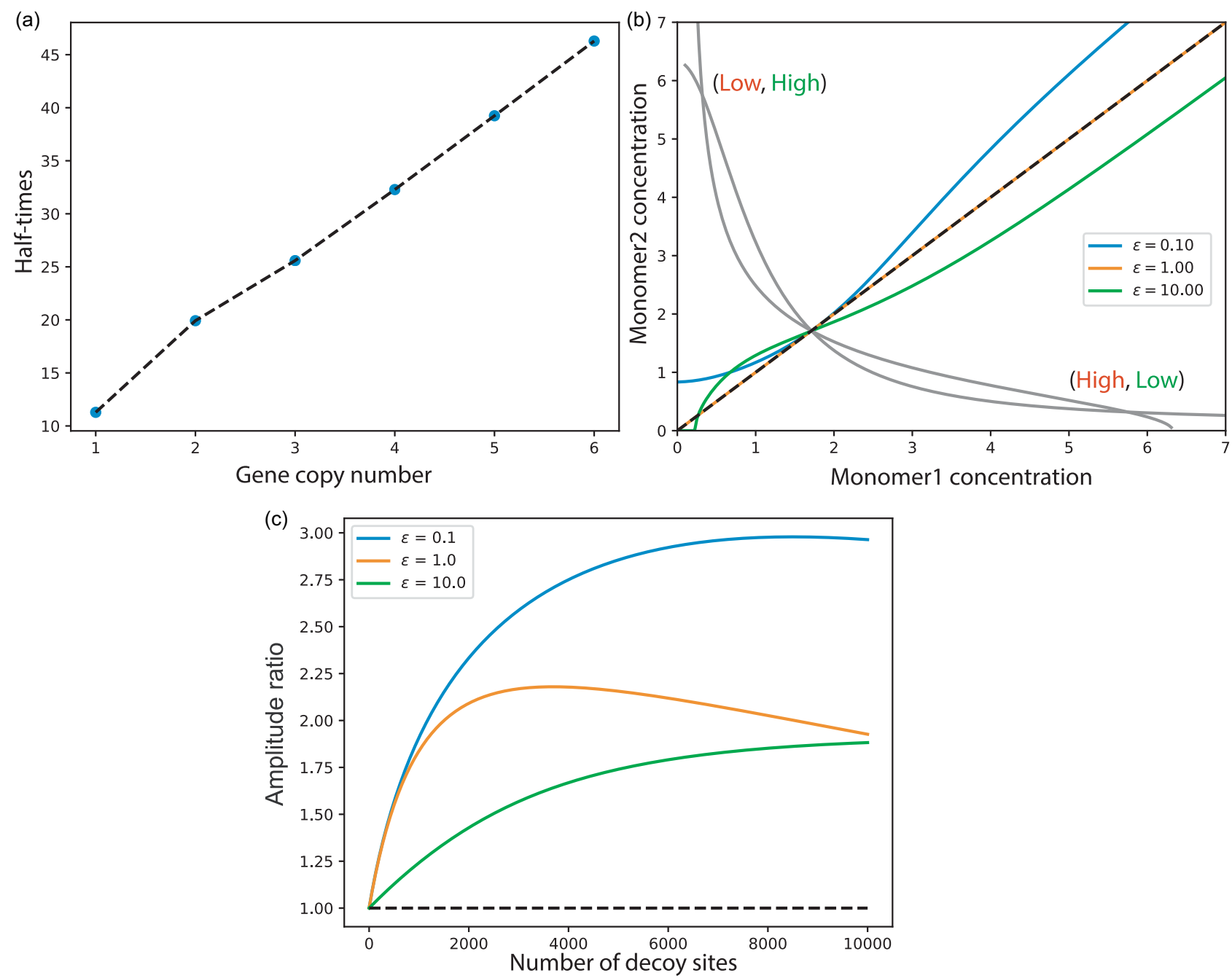

FIG. 8. (a) The curves show a linear increase in half-times to reach the steady state with increase in gene copy number $N_{p}$. For computational reliability, these half-times are computed by averaging over an ensemble of 100 random initial conditions chosen in the neighborhood of steady state. (b) Separatrixes have been plotted for $N_{p}=1$ for different protein binding affinities of decoys. The curve's change is significant and therefore, basins of attraction of the two stables states are considerably altered. (c) Amplitude ratio is highly sensitive to variation in protein binding affinities of decoys for $N_{p}=1$. The effect appears to be suppressed for high binding affinities.

The prefactors are

$$
\begin{aligned}
& p\left(x_{1}\right)=1+4 r x_{1}+\frac{4 \tilde{N}_{p} x_{1}}{\left(1+x_{1}^{2}\right)^{2}}+\frac{4 \tilde{N}_{d} \epsilon x_{1}}{\left(1+\epsilon x_{1}^{2}\right)^{2}} \\
& p\left(x_{2}\right)=1+4 r x_{2}+\frac{4 \tilde{N}_{p} x_{2}}{\left(1+x_{2}^{2}\right)^{2}}+\frac{4 \tilde{N}_{d} \epsilon x_{2}}{\left(1+\epsilon x_{2}^{2}\right)^{2}} .
\end{aligned}
$$

In the equations above, we assumed the rate of binding and unbinding of protein dimers to decoys sites to be equal for both genes, $\bar{c}_{d, 1}=\bar{c}_{d, 2}\left(=\bar{c}_{d}\right)$, which implies $\epsilon_{1}=\epsilon_{2}(=\epsilon)$.

Now this symmetry of decoy sites is effectively broken by including two different species of decoys such that $N_{d, 1} \neq$ $N_{d, 2}$. It modifies prefactors in Eqs. (B5) as follows:

$$
\begin{aligned}
& p\left(x_{1}\right)=1+4 r x_{1}+\frac{4 \tilde{N}_{p} x_{1}}{\left(1+x_{1}^{2}\right)^{2}}+\frac{4 \tilde{N}_{d_{1}} x_{1}}{\left(1+\eta x_{1}^{2}\right)^{2}} \\
& p\left(x_{2}\right)=1+4 r x_{2}+\frac{4 \tilde{N}_{p} x_{2}}{\left(1+x_{2}^{2}\right)^{2}}+\frac{4 \tilde{N}_{d_{2}} x_{2}}{\left(1+x_{2}^{2}\right)^{2}} .
\end{aligned}
$$

For simplicity of analysis, we assume $\epsilon_{1}=\epsilon_{2}=1$. The main text shows the influence of $\tilde{N}_{d, 1}<\tilde{N}_{d, 2}$ on the basin of attraction of the two stable fixed points at saddle node bifurcation.

\section{Basin of attraction for different protein binding affinities of decoys}

As noted in the main text, an alternative way to heterogeneous decoys is to consider identical decoy number with different protein binding affinities $\epsilon_{1} \neq \epsilon_{2}$. We find the separatrix sensitive to this change, and as a consequence, the basin of attraction of stable states (high, low) and (low, high) demonstrates considerable variation [see Fig. 8(b)]. This appears to be a nontrivial behavior and warrants deeper investigation; we leave it for future research.

\section{Parameters}

We use the following parameter values to perform computations: 


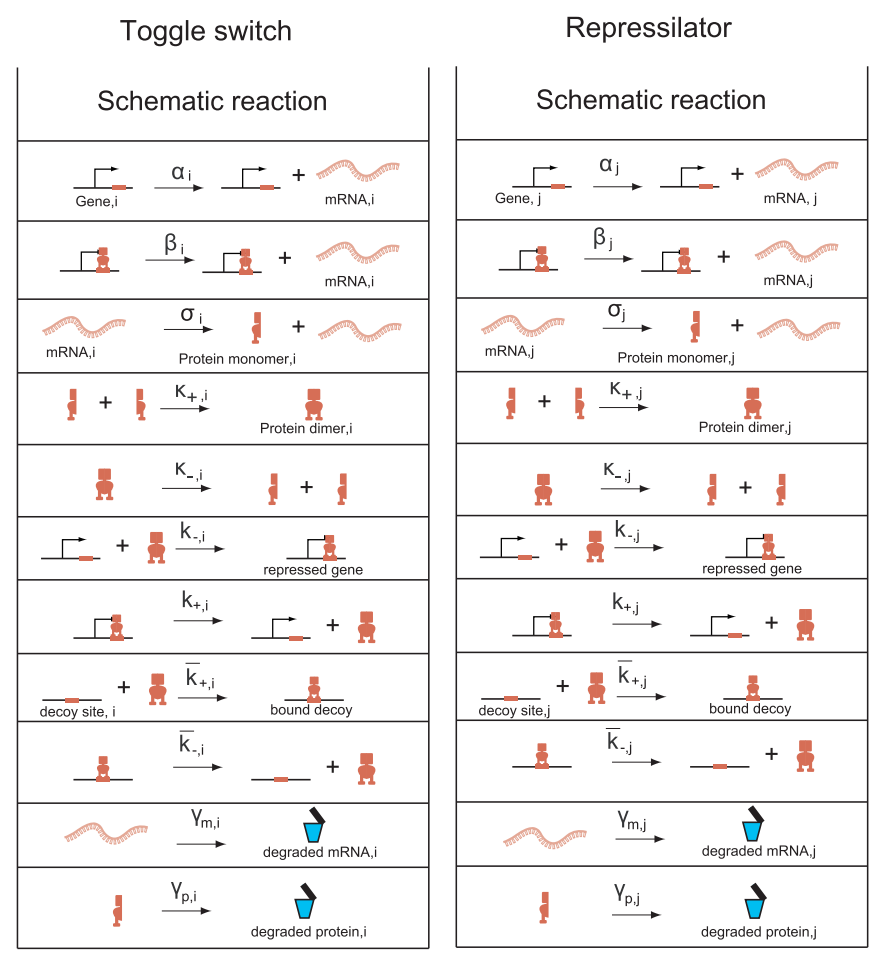

FIG. 9. List of reactions used for the toggle switch and repressilator systems. For the toggle switch, $i$ takes values 1 and 2, corresponding to two genes of the motif. For the repressilator, $j$ takes values 1,2 , and 3 , corresponding to three genes of the motif.

$C=10^{-9} \mathrm{M}, \quad c_{p}=10^{7} \mathrm{M}^{-1}, \quad c_{d}=10^{7} \mathrm{M}^{-1}, \quad \bar{c}_{d}=$ $10^{7} \mathrm{M}^{-1}, \sigma=0.5$.

Basin of attraction and $\alpha \approx 6.32 \mathrm{~min}^{-1}$,

time evolution of $\quad \beta \approx 0.14 \mathrm{~min}^{-1}, \gamma_{p}=0.05 \mathrm{~min}^{-1}$,

monomer $\quad \gamma_{m}=0.10 \mathrm{~min}^{-1}\left(\Gamma=0.5, \kappa_{1}=632.50\right.$,

concentration as in $\quad$ and $\left.\kappa_{2}=13.75\right)[30,37,41]$.

Figs. 3 and 4:

\section{APPENDIX C: REPRESSILATOR}

We use Eqs. (A15) with $\beta=0$ (no transcription from bound promoter sites) to get the model equations for the repressilator:

$$
\begin{gathered}
p\left(x_{i}\right) \dot{x}_{i}=\sigma m_{i}-\gamma_{p} x_{i} \\
\dot{m}_{i}=\frac{\alpha C N_{p}}{1+c_{p} c_{d} x_{k}^{2}}-\gamma_{m} m_{i},
\end{gathered}
$$

where $i \in\{1,2,3\}, k \in\{3,1,2\}$. The full set of reactions are given in Fig. 9. Rescalings: $\gamma_{m} t \rightarrow t, \sqrt{c_{d} c_{p}} x_{i} \rightarrow x_{i}$, and $\left(\sigma \sqrt{c_{d} c_{p}}\right) /\left(\gamma_{m} \beta\right) m_{i} \rightarrow m_{i}$, to obtain

$$
\begin{gathered}
p\left(x_{i}\right) \dot{x}_{i}=-\Gamma\left(x_{i}-m_{i}\right) \\
\dot{m}_{i}=\frac{\kappa \tilde{N}_{p}}{1+x_{k}^{2}}-m_{i},
\end{gathered}
$$

where $\Gamma=\gamma_{p} / \gamma_{m}, \kappa=\alpha \sigma /\left(\gamma_{m} \gamma_{p}\right), \tilde{N}_{p}=\sqrt{c_{d} c_{p}} C N_{p}$. The prefactor is given by

$$
p\left(x_{i}\right)=1+4 r x_{i}+\frac{4 \tilde{N}_{p} x_{i}}{\left(1+x_{i}^{2}\right)^{2}}+\frac{4 \tilde{N}_{d} \epsilon x_{i}}{\left(1+\epsilon x_{i}^{2}\right)^{2}},
$$

where $r=\sqrt{c_{p} / c_{d}}$ and $\epsilon=\overline{c_{d}} / c_{d}, \tilde{N}_{d}=\sqrt{c_{d} c_{p}} C N_{d}$.

\section{Stability analysis and Hopf frequency}

The repressilator defined by Eqs. (C3) has a symmetric equilibrium $S=\left(x_{i}, m_{i}\right)=(\bar{x}, \bar{x})$, which is a unique real solution of the equation:

$$
\bar{x}+\bar{x}^{3}=\kappa \tilde{N}_{p}
$$

The Jacobian of the system around this equilibrium reads

$$
J=\left[\begin{array}{cccccc}
-\frac{\Gamma}{p(\bar{x})} & \frac{\Gamma}{p(\bar{x})} & 0 & 0 & 0 & 0 \\
0 & -1 & 0 & 0 & -\frac{2 \bar{x}^{3}}{\kappa \tilde{N}_{p}} & 0 \\
0 & 0 & -\frac{\Gamma}{p(\bar{x})} & \frac{\Gamma}{p(\bar{x})} & 0 & 0 \\
-\frac{2 \bar{x}^{3}}{\kappa \tilde{N}_{p}} & 0 & 0 & -1 & 0 & 0 \\
0 & 0 & 0 & 0 & -\frac{\Gamma}{p(\bar{x})} & \frac{\Gamma}{p(\bar{x})} \\
0 & 0 & -\frac{2 \bar{x}^{3}}{\kappa \tilde{N}_{p}} & 0 & 0 & -1
\end{array}\right]
$$

The Jacobian $J$ has the following eigenvalues at the symmetric equilibrium defined above:

$$
\lambda_{1,2,3,4}=-\frac{\rho}{2}-\frac{1}{2} \pm \sqrt{\frac{\rho^{2}}{4}-\frac{\rho \chi}{2} \pm i \frac{\sqrt{3} \rho \chi}{2}-\frac{\rho}{2}+\frac{1}{4}}
$$

$$
\lambda_{5,6}=-\frac{\rho}{2}-\frac{1}{2} \pm \sqrt{\frac{\rho^{2}}{4}+\rho \chi-\frac{\rho}{2}+\frac{1}{4}}
$$

where $\rho=\frac{\Gamma}{p(\bar{x})}$ and $\chi=-\frac{2 \bar{x}^{3}}{\kappa \tilde{N}_{p}}$.

\section{a. Bifurcation analysis: Hopf Frequency}

When a complex eigenvalue $\lambda$ crosses the imaginary axis, we get the Hopf bifurcation. At the point of bifurcation, the real part of an eigenvalue becomes zero and the imaginary part gives the frequency of oscillations.

Taking $\lambda_{1}=-\frac{\rho}{2}-\frac{1}{2} \pm \sqrt{\frac{\rho^{2}}{4}-\frac{\rho \chi}{2} \pm i \frac{\sqrt{3} \rho \chi}{2}-\frac{\rho}{2}+\frac{1}{4}}$, we write $\lambda_{1}$ as follows:

$$
\lambda_{1}=-\frac{\rho}{2}-\frac{1}{2}+\mu+i \omega
$$

Setting the real part to zero, we get $\mu=\frac{\rho+1}{2}$. But $\mu+i \omega$ is given by

$$
(\mu+i \omega)^{2}=\frac{\rho^{2}}{4}-\frac{\rho \chi}{2} \pm i \frac{\sqrt{3} \rho \chi}{2}-\frac{\rho}{2}+\frac{1}{4} .
$$



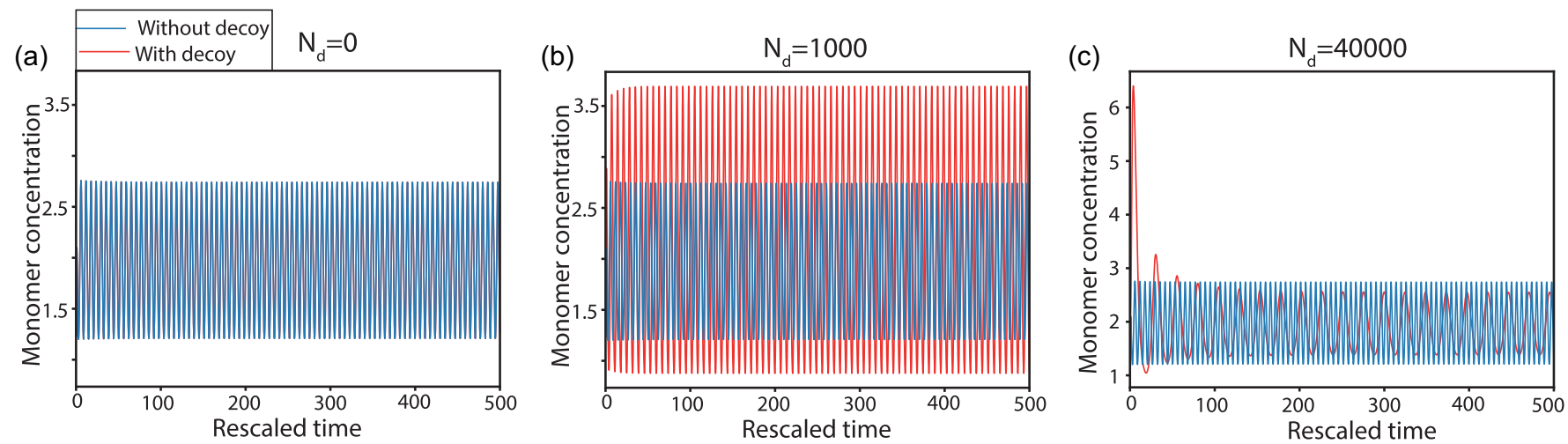

FIG. 10. Examples to show variation in amplitude of monomer concentration with time for different numbers of decoy sites at $N=1$. The blue curve indicates the variation in the absence of decoy sites, while the red curve shows that in the presence of decoy sites. (a) The case for the absence decoys, i.e., $N_{d}=0.0$. (b) For $N_{d}=1000$, amplitude of oscillation increases. (c) Amplitude decreases at $N_{d}=40000$.

Equating the real and imaginary parts, we find, for the imaginary part,

$$
2 \mu \omega=\frac{\sqrt{3} \rho \chi}{2} .
$$

Substituting the expression for $\mu$,

$$
\omega=\frac{\sqrt{3} \chi}{2} \frac{\rho}{\rho+1} .
$$

Substituting the expressions for $\rho$ and $\chi$, this imaginary part gives the Hopf frequency,

$$
\omega=\frac{\sqrt{3} \bar{x}^{3}}{\kappa \tilde{N}_{p}} \frac{\Gamma}{\Gamma+p(\bar{x})},
$$

where the prefactor is given by

$$
p(\bar{x})=1+4 r \bar{x}+\frac{4 \tilde{N}_{p} \bar{x}}{\left(1+\bar{x}^{2}\right)^{2}}+\frac{4 \tilde{N}_{d} \epsilon \bar{x}}{\left(1+\epsilon \bar{x}^{2}\right)^{2}} .
$$

\section{b. Bifurcation analysis: Ratio of frequency}

We define $\omega_{r}$ to the ratio of Hopf frequencies with and without decoys for a given $N_{p}$ ( $\omega$ and $\omega_{0}$, respectively) while other parameters are equal. Using Eq. (C10) we get

$$
\omega_{r}=\frac{\omega}{\omega_{0}}=\frac{\Gamma+p_{0}(\bar{x})}{\Gamma+p(\bar{x})} .
$$

\section{c. Bifurcation analysis: Condition for change in stability}

The equilibrium point of the system will change its stability when the first pair of complex eigenvalues crosses the imaginary axis. The point thus looses it stability and a limit cycle occurs via Hopf bifurcation. We continue with Eq. (C9) and equate the real part to obtain

$$
\mu^{2}-\omega^{2}=\frac{\rho^{2}}{4}-\frac{\rho \chi}{2}-\frac{\rho}{2}+\frac{1}{4} .
$$

After substituting the expressions for $\mu$ and $\omega$, a few steps of algebra lead to the following expression:

$$
\frac{(\rho+1)^{2}}{\rho}=\frac{3 \chi^{2}}{4+2 \chi} .
$$

We again substitute the known expressions and identify $\Gamma$ here as the critical $\Gamma_{c}$, and then we find

$$
\frac{\left[\Gamma_{c}+p(\bar{x})\right]^{2}}{\Gamma_{c} p(\bar{x})}=\frac{3 \chi^{2}}{4+2 \chi}
$$

with $\chi=-\frac{2 \bar{x}^{3}}{\kappa \tilde{N}_{p}}$. Here Eq. (C12) is quadratic in $\Gamma$ and hence generates two branches of the solution manifold, dividing the parameter space in stable and unstable regions.

\section{Parameters}

We use the following parameters to perform analysis: $C=$ $10^{-9} \mathrm{M}, c_{p}=10^{7} \mathrm{M}^{-1}, c_{d}=10^{7} \mathrm{M}^{-1}, \bar{c}_{d}=10^{7} \mathrm{M}^{-1}, \sigma=$ 0.5 .

\section{Time evolution of monomer} concentration Fig. 4(b).

Time evolution of monomer concentration Figs. 4(c)-4(d).

Frequency ratio and amplitude ratio variation Fig. 6.

$$
\begin{aligned}
& \alpha=9.60 \mathrm{~min}^{-1}, \\
& \gamma_{p}=0.08 \mathrm{~min}^{-1}, \\
& \gamma_{m}=0.10 \mathrm{~min}^{-1}(\Gamma=0.80 \text { and } \\
& \kappa=600.0)[30,37,41] . \\
& \alpha \approx 1.40 \mathrm{~min}^{-1}, \\
& \gamma_{p}=0.24 \mathrm{~min}^{-1}, \\
& \gamma_{m}=0.02 \mathrm{~min}^{-1}(\Gamma=12.0 \text { and } \\
& \kappa=145.0)[30,37,41] . \\
& \alpha=6.40 \mathrm{~min}^{-1}, \\
& \gamma_{p}=0.40 \mathrm{~min}^{-1}, \\
& \gamma_{m}=0.01 \mathrm{~min}^{-1}(\Gamma=40.0 \text { and } \\
& \kappa=800.0)[30,37,41] .
\end{aligned}
$$

[1] E. H. Davidson, The Regulatory Genome: Gene Regulatory Networks in Development and Evolution, 1st ed. (Academic Press, Burlington, MA, 2006).
[2] E. H. Davidson, J. P. Rast, P. Oliveri, A. Ransick, C. Calestani, C.-H. Yuh, T. Minokawa, G. Amore, V. Hinman, C. ArenasMena, O. Otim, C. T. Brown, C. B. Livi, P. Y. Lee, R. Revilla, 
A. G. Rust, Z. j. Pan, M. J. Schilstra, P. J. C. Clarke, M. I. Arnone et al., Science 295, 1669 (2002).

[3] E. H. Davidson, Nature (London) 468, 911 (2010).

[4] S. S. Shen-Orr, R. Milo, S. Mangan, and U. Alon, Nat. Genet. 31, 64 (2002).

[5] T.-H. Lee and N. Maheshri, Mol. Syst. Biol. 8, 576 (2012).

[6] H.-W. Ma, B. Kumar, U. Ditges, F. Gunzer, J. Buer, and A.-P. Zeng, Nucleic Acids Res. 32, 6643 (2004).

[7] L. Potvin-Trottier, N. D. Lord, G. Vinnicombe, and J. Paulsson, Nature (London) 538, 514 (2016).

[8] T. S. Gardner, C. R. Cantor, and J. J. Collins, Nature (London) 403, 339 (2000).

[9] J. Stricker, S. Cookson, M. R. Bennett, W. H. Mather, L. S. Tsimring, and J. Hasty, Nature (London) 456, 516 (2008).

[10] M. B. Elowitz and S. Leibler, Nature (London) 403, 335 (2000).

[11] G. P. Wagner, M. Pavlicev, and J. M. Cheverud, Nat. Rev. Genet. 8, 921 (2007).

[12] S. M. Lyons, W. Xu, J. Medford, and A. Prasad, PLoS Comput. Biol. 10, e1003533 (2014).

[13] M. T. Cooling, V. Rouilly, G. Misirli, J. Lawson, T. Yu, J. Hallinan, and A. Wipat, Bioinformatics 26, 925 (2010).

[14] T. Shimada, T. Kawai, K. Takeda, M. Matsumoto, J. Inoue, Y. Tatsumi, A. Kanamaru, and S. Akira, Int. Immunol. 11, 1357 (1999).

[15] S. C. Gupta, C. Sundaram, S. Reuter, and B. B. Aggarwal, Biochim. Biophys. Acta 1799, 775 (2010).

[16] A. Antonaki, C. Demetriades, A. Polyzos, A. Banos, G. Vatsellas, M. D. Lavigne, E. Apostolou, E. Mantouvalou, D. Papadopoulou, G. Mosialos, and D. Thanos, J. Biol. Chem. 286, 38768 (2011).

[17] B. Zhao, L. A. Barrera, I. Ersing, B. Willox, S. C. Schmidt, H. Greenfeld, H. Zhou, S. B. Mollo, T. T. Shi, K. Takasaki, S. Jiang, E. Cahir-McFarland, M. Kellis, M. L. Bulyk, E. Kieff, and B. E. Gewurz, Cell Rep. 8, 1595 (2014).

[18] Z. Wang, A. L. Martins, and C. G. Danko, Bioinformatics 32, 3024 (2016).

[19] J. S. Beckmann, X. Estivill, and S. E. Antonarakis, Nat. Rev. Genet. 8, 639 (2007).

[20] G. M. Cooper, Regulation of Transcription in Eukaryotes (ASM Press, Washington, D.C., Sunderland, Massachusetts, 2004), 3rd ed.

[21] R. Redon, S. Ishikawa, K. R. Fitch, L. Feuk, G. H. Perry, T. D. Andrews, H. Fiegler, M. H. Shapero, A. R. Carson, W. Chen, E. K. Cho, S. Dallaire, J. L. Freeman, J. R. González, M. Gratacòs, J. Huang, D. Kalaitzopoulos, D. Komura, J. R. MacDonald, C. R. Marshall et al., Nature (London) 444, 444 (2006).

[22] B. E. Stranger, M. S. Forrest, M. Dunning, C. E. Ingle, C. Beazley, N. Thorne, R. Redon, C. P. Bird, A. de Grassi, C. Lee, C. Tyler-Smith, N. Carter, S. W. Scherer, S. Tavaré, P. Deloukas, M. E. Hurles, and E. T. Dermitzakis, Science 315, 848 (2007).

[23] J. M. Kidd, G. M. Cooper, W. F. Donahue, H. S. Hayden, N. Sampas, T. Graves, N. Hansen, B. Teague, C. Alkan, F. Antonacci, E. Haugen, T. Zerr, N. A. Yamada, P. Tsang, T. L. Newman, E. Tüzün, Z. Cheng, H. M. Ebling, N. Tusneem, R. David et al., Nature (London) 453, 56 (2008).

[24] N. J. Guido, X. Wang, D. Adalsteinsson, D. McMillen, J. Hasty, C. R. Cantor, T. C. Elston, and J. J. Collins, Nature (London) 439, 856 (2006).
[25] H. Bremer, P. Dennis, and M. Ehrenberg, Biochimie 85, 597 (2003).

[26] R. L. Wang, A. Stec, J. Hey, L. Lukens, and J. Doebley, Nature (London) 398, 236 (1999).

[27] A. Navarro-Quezada and D. J. Schoen, Proc. Natl. Acad. Sci. USA 99, 268 (2002).

[28] S. E. Luria and R. Dulbecco, Genetics 34, 93 (1949).

[29] M. Kellis, B. W. Birren, and E. S. Lander, Nature (London) 428, 617 (2004).

[30] Y. Mileyko, R. I. Joh, and J. S. Weitz, Proc. Natl. Acad. Sci. USA 105, 16659 (2008).

[31] T. W. Grunberg and D. Del Vecchio, Curr. Opin. Biotechnol. 63, 41 (2020).

[32] A. Burger, A. M. Walczak, and P. G. Wolynes, Proc. Natl. Acad. Sci. USA 107, 4016 (2010).

[33] M. Z. Ali, V. Parisutham, S. Choubey, and R. C. Brewster, Elife 9, e56517 (2020).

[34] D. Das, S. Dey, R. C. Brewster, and S. Choubey, PLoS Comput. Biol. 13, e1005491 (2017).

[35] S. Jayanthi, K. S. Nilgiriwala, and D. Del Vecchio, ACS Synth. Biol. 2, 431 (2013).

[36] R. Hermsen, D. W. Erickson, and T. Hwa, PLoS Comput. Biol. 7, e1002265 (2011).

[37] U. Alon, An Introduction to Systems Biology: Design Principles of Biological Circuits, 1st ed. (Chapman and Hall/CRC, Boca Raton, FL, 2006).

[38] T. Tian and K. Burrage, J. Theor. Biol. 227, 229 (2004).

[39] J. Jaruszewicz-Błońska and T. Lipniacki, BMC Syst. Biol. 11, 117 (2017).

[40] A. Lipshtat, A. Loinger, N. Q. Balaban, and O. Biham, Phys. Rev. Lett. 96, 188101 (2006).

[41] M. R. Bennett, D. Volfson, L. Tsimring, and J. Hasty, Biophys. J. 92, 3501 (2007).

[42] F. Bertaux, S. Marguerat, and V. Shahrezaei, R. Soc. Open Sci. 5172234 (2018).

[43] B. Kaity, R. Sarkar, B. Chakrabarti, and M. K. Mitra, Sci. Rep. 8, 7358 (2018).

[44] T. Tian and K. Burrage, Proc. Natl. Acad. Sci. USA 103, 8372 (2006).

[45] E. M. Ozbudak, M. Thattai, H. N. Lim, B. I. Shraiman, and A. Van Oudenaarden, Nature (London) 427, 737 (2004).

[46] M. Santillán, M. C. Mackey, and E. S. Zeron, Biophys. J. 92, 3830 (2007).

[47] W. Xiong and J. E. Ferrell, Nature (London) 426, 460 (2003).

[48] C. P. Bagowski and J. E. Ferrell, Curr. Biol. 11, 1176 (2001).

[49] A. Harding, T. Tian, E. Westbury, E. Frische, and J. F. Hancock, Curr. Biol. 15, 869 (2005).

[50] N. I. Markevich, J. B. Hoek, and B. N. Kholodenko, J. Cell Biol. 164, 353 (2004).

[51] J. Garcia-Ojalvo, M. B. Elowitz, and S. H. Strogatz, Proc. Natl. Acad. Sci. USA 101, 10955 (2004).

[52] Q.-1. Dong, X.-y. Xing, Y. Han, X.-1. Wei, and S. Zhang, CellBio 9, 29 (2020).

[53] M. R. Doyle, S. J. Davis, R. M. Bastow, H. G. McWatters, L. Kozma-Bognár, F. Nagy, A. J. Millar, and R. M. Amasino, Nature (London) 419, 74 (2002). 
[54] L. Wu, Q. Ouyang, and H. Wang, PLoS ONE 12, e0171263 (2017).

[55] D. H. Nagel and S. A. Kay, Curr. Biol. 22, R648 (2012).

[56] A. Pokhilko, A. P. Fernández, K. D. Edwards, M. M. Southern, K. J. Halliday, and A. J. Millar, Mol. Syst. Biol. 8, 574 (2012).

[57] R. C. Brewster, F. M. Weinert, H. G. Garcia, D. Song, M. Rydenfelt, and R. Phillips, Cell 156, 1312 (2014).
[58] M. Khammash, BMC Biol. 14, 22 (2016).

[59] L. Poliseno, L. Salmena, J. Zhang, B. Carver, W. J. Haveman, and P. P. Pandolfi, Nature (London) 465, 1033 (2010).

[60] Y. Tay, J. Rinn, and P. P. Pandolfi, Nature (London) 505, 344 (2014).

[61] See https://github.com/SwetamberD/Network-motif. 